\title{
Uncertainty budgets of major ozone absorption cross sections used in UV remote sensing applications
}

\author{
Mark Weber ${ }^{1}$, Victor Gorshelev ${ }^{1}$, and Anna Serdyuchenko ${ }^{1, \mathrm{a}}$ \\ ${ }^{1}$ Institut für Umweltphysik, Universität Bremen FB1, P.O. Box 330 440, 28334 Bremen, Germany \\ ${ }^{a}$ now at: OHB System AG, Manfred-Fuchs-Straße 1, 82234 Weßling, Germany \\ Correspondence to: Mark Weber (weber@uni-bremen.de)
}

Received: 2 February 2016 - Published in Atmos. Meas. Tech. Discuss.: 14 April 2016

Revised: 10 August 2016 - Accepted: 16 August 2016 - Published: 8 September 2016

\begin{abstract}
Detailed uncertainty budgets of three major ultraviolet (UV) ozone absorption cross-section datasets that are used in remote sensing application are provided and discussed. The datasets are Bass-Paur (BP), Brion-DaumontMalicet (BDM), and the more recent SerdyuchenkoGorshelev (SG). For most remote sensing application the temperature dependence of the Huggins ozone band is described by a quadratic polynomial in temperature (BassPaur parameterization) by applying a regression to the crosssection data measured at selected atmospherically relevant temperatures. For traceability of atmospheric ozone measurements, uncertainties from the laboratory measurements as well as from the temperature parameterization of the ozone cross-section data are needed as input for detailed uncertainty calculation of atmospheric ozone measurements. In this paper the uncertainty budgets of the three major ozone cross-section datasets are summarized from the original literature. The quadratic temperature dependence of the crosssection datasets is investigated. Combined uncertainty budgets is provided for all datasets based upon Monte Carlo simulation that includes uncertainties from the laboratory measurements as well as uncertainties from the temperature parameterization. Between 300 and $330 \mathrm{~nm}$ both BDM and SG have an overall uncertainty of $1.5 \%$, while BP has a somewhat larger uncertainty of $2.1 \%$. At temperatures below about $215 \mathrm{~K}$, uncertainties in the BDM data increase more strongly than the others due to the lack of very low temperature laboratory measurements (lowest temperature of BDM available is $218 \mathrm{~K}$ ).
\end{abstract}

\section{Introduction}

The three ozone absorption cross sections in common use for many remote sensing applications are the Bass-Paur (BP) data (Bass and Paur, 1985; Paur and Bass, 1985), the Brion-Daumont-Malicet (BDM) data (Daumont et al., 1992; Brion et al., 1993; Malicet et al., 1995), and the very recent Serdyuchenko-Gorshelev (SG) data (Gorshelev et al., 2014; Serdyuchenko et al., 2011, 2014). While the data from BDM and SG are absolute cross-section measurements, the BP data were scaled to the so-called Hearn value at the mercury line wavelength $(253.65 \mathrm{~nm})$. The standard retrievals applied to the ground Brewer and Dobson spectrophotometer data use the BP data (e.g., Redondas et al., 2014), while the satellite community uses any of the three datasets when retrieval is limited to the ultraviolet (UV) spectral region (WMO-GAW, 2015; ACSO, 2010; Orphal et al., 2016).

For the review of uncertainties, original publications reporting on results of the experimental work were considered first. Since BP data were absolutely scaled using Hearn data (Hearn, 1961), the latter is also included in this review. There is a lack of consistency in the presentation of measurement uncertainty budgets across different papers. An attempt to analyze and harmonize the reported uncertainties is made in the following sections. In some cases not all measured quantities were reported and in most cases detailed description of the data processing procedures is missing. It is very likely that the published measurement uncertainties are incomplete and the overall uncertainties are thus underestimated.

In this paper we use a Monte Carlo simulation (JCGM101,2008 ) in order to get a better estimate of uncertainties in the major ozone absorption cross-section data. This method 
will allow us to combine the uncertainties from the laboratory measurements with those from the temperature parameterization that is widely used to interpolate between the laboratory temperatures. The temperature dependence in the UV ozone absorption cross section is usually expressed as a quadratic polynomial (Bass-Paur parameterization). Having only four to five temperatures available as in the case of BDM and BP, a quadratic polynomial will, at many wavelengths, overfit the data (perfect matches). However, in the Monte Carlo (MC) simulation that includes uncertainties from the laboratory measurements, more realistic contributions from uncertainties in the temperature parameterization to the overall uncertainty will be obtained.

In this paper we start with a brief summary of measurement principles in the laboratory (Sect. 2), followed by a review of the uncertainties of the UV ozone cross-section data (Sect. 3). In Sect. 4 the temperature dependence in the three major ozone cross-section datasets is discussed followed by Sect. 5, which summarizes the Monte Carlo simulation to obtain the overall uncertainty budgets of the major datasets. Section 6 provides a summary and conclusion.

\section{Measurement technique}

Ozone absorption cross sections are produced by performing spectroscopic measurements and subsequent analysis to convert the recorded spectra into absorption cross sections in units of $\mathrm{cm}^{2}$ molecule ${ }^{-1}$. The absorption spectroscopy is based on the Beer-Lambert law, which describes the attenuation of the light intensity transmitted through the absorbing medium.

For gaseous species the Beer-Lambert law can be written as

$I(\lambda)=I_{0}(\lambda) e^{-\int_{0}^{L} n(T, p, l) \cdot \sigma(\lambda, T) \mathrm{d} l}$.

Here, $I_{0}(\lambda)$ is the light intensity in the absence of absorbing molecules (background), $n$ is the absorbing gas number density, which is generally a function of temperature $T$, pressure $p$, and the position $l$ along the beam path. $L$ is the total absorption path length and $\sigma\left(\mathrm{cm}^{2}\right.$ molecule $\left.{ }^{-1}\right)$ is the wavelength-dependent (and normally also temperaturedependent) absorption cross section.

In a laboratory environment it is possible to control the experimental conditions with sufficient precision, so that the number density $n\left(\mathrm{~cm}^{-3}\right)$ is assumed to be homogeneously distributed along the absorption path of a known length $L$ (cm). From measurements of other parameters, such as $T(\mathrm{~K})$ and $p(\mathrm{~Pa})$, the value of $n$ is calculated assuming the ideal gas law $p=n \cdot k_{\mathrm{B}} \cdot T\left(k_{\mathrm{B}}\right.$, Boltzmann constant). In this case Eq. (1) can be transformed to

$$
A(\lambda, T)=1-I / I_{0}=1-e^{-\sigma(\lambda, T) \cdot n \cdot L},
$$

where $A$ is the unitless absorbance. The unitless optical density (OD) is then expressed as

$\mathrm{OD}(\lambda, T)=\ln \left(I / I_{0}\right)=e^{-\sigma(\lambda, T) \cdot n \cdot L}$.

The Beer-Lambert law, used to describe the absorption, is applicable when the density of the absorbing medium is low enough to avoid nonlinear effects, the medium itself does not scatter light, and exposure to light does not change the properties of the medium. The first two points are valid for gaseous media, like the ozone sample collected in the experimental cell. Exposure to UV radiation leads to photolysis of ozone and causes an observable change in ozone concentration if measurement time is long enough.

Absolute cross sections can be derived from the optical density if species concentration, temperature, and absorption path length are known. Uncertainties of cross sections derived from Eq. (3) are influenced by measurement uncertainties of these contributing parameters. Since ozone is a reactive and highly explosive gas, many measurements were done using a flow of oxygen / ozone mixture, where the partial pressure of ozone is unknown and values from other published absolute ozone cross-section measurements are used to find a scaling factor that converts the measured optical density into absorption cross section (e.g., Chehade et al., 2013a, b).

Absolute measurements performed at selected wavelengths, with special attention given to the control of the experimental parameters, are often used to calibrate the relative cross sections (optical densities). The latter was done for the BP data (Bass and Paur, 1985; Paur and Bass, 1985) as all their relative spectra were scaled to the ozone absorption cross-section value at $253.65 \mathrm{~nm}$ (mercury line) as reported by Hearn (1961). Thus the uncertainties in the reference data propagate into the calibrated spectra.

Depending on the kind of spectrometer used for broadband (Fourier transform, grating) or single wavelength measurements, registered spectra are inevitably subject to multiple sources of uncertainties - stochastic intensity variations caused by detector noise, light source intensity fluctuations etc. (JCGM-100, 2008). Spectral random error can be characterized by the signal-to-noise ratio $(S / N)$, and one of the ways to improve the quality of the measurements is acquisition of multiple spectra obtained under repeatable conditions (JCGM-100, 2008). Uncertainty of the resulting average value is then represented by the standard deviation of the mean.

Spectrometers are characterized by the spectral resolution and wavelength calibration to some reference values, which influences the wavelength uncertainty of the produced data. For example, dispersion-based instruments can be wavelength-calibrated using isolated atomic emission lines of $\mathrm{Hg}$ or $\mathrm{Cd}$ lamps, and Fourier transform spectrometers are auto-calibrated with the built-in stabilized $\mathrm{He}-\mathrm{Ne}$ laser. 
Instrumental uncertainties of other measured quantities temperature $T$, ozone (partial) pressure $p$, absorption path length $L$ also contribute to the total absorption cross-section uncertainty. For broadband laboratory measurements covering a large wavelength range, like the Hartley-Huggins band of ozone, cross-section values change by up to 7 orders of magnitude; therefore optical density spectra are recorded using different combination of cell lengths and partial pressures (e.g., Gorshelev et al., 2014; Serdyuchenko et al., 2014). The various spectra are then concatenated to cover the entire spectral range, which leads to additional uncertainties.

\section{Review of reported uncertainties}

\subsection{Uncertainty budget of Hearn}

Dating back to 1961, Hearn reported on ozone absorption cross sections at six selected wavelengths, of which the value at $\lambda=253.65 \mathrm{~nm}$ is of particular interest, since it has been measured in many studies and is considered a standard reference (see Viallon et al. (2015) and references therein). The following information of the laboratory measurements by Hearn (1961) is known:

- spectral resolution: $0.09 \mathrm{~nm}(\mathrm{Hg}$ emission line width at $253.65 \mathrm{~nm})$;

- temperature: $295 \mathrm{~K}$;

- temperature uncertainty: not reported ("Errors due to the variation of the temperature of the ozone/oxygen mixture during the experiment are quite negligible; the apparatus was housed in a cellar, the temperature of which was thermostatically controlled.");

- absolute scaling: pressure observation of the pure $\mathrm{O}_{3} \rightarrow \mathrm{O}_{2}$ decomposition.

Table 1 provides the original notation of uncertainty budget from Hearn (1961). The uncertainties are divided into type A and type B uncertainties (JCGM-100, 2008). Type A uncertainty means that it is derived from statistical analysis and an observed frequency distribution. Type B uncertainties are not derived from statistical analysis, and a probability distribution is assumed that is based upon past experiences or is derived from external specifications. The breakdown of evaluation type of the uncertainties is also shown in Table 1.

Little to no detail is provided on the accuracy of the instruments used during measurements. Given the reported $\pm 2.4 \times 10^{-19} \mathrm{~cm}^{2}$ interval around the $114.7 \times 10^{-19} \mathrm{~cm}^{2}$ value of absorption cross section, and assuming a normal distribution of possible values (JCGM-100, 2008), the relative standard measurement uncertainty of the ozone absorption cross section at $253.65 \mathrm{~nm}$ is estimated to be around $2 \%$. It should be noted here that more recent measurements (Viallon et al., 2015;) indicate lower values for the mercury line that lies about 1.4 to $1.8 \%$ lower than Hearn's value, but is within the uncertainty of the Hearn's experiment. Compared to all available measurements reported, Hearn's value is close to the upper range of values (e.g., Sofen et al., 2015; WMOGAW, 2015).

\subsection{Uncertainty budget of BP}

The team of Bass and Paur (Bass and Paur, 1985; Paur and Bass, 1985) provided cross-section data for a broad spectral range and at several temperatures. The following information is available from the BP data:

- wavelength range: $245-340 \mathrm{~nm}$;

- spectral resolution: $0.05 \mathrm{~nm}$;

- wavelength grid: $0.05 \mathrm{~nm}$;

- wavelength calibration: 23 points between 200 and $365 \mathrm{~nm}$ (Hg, Cd, Zn lines);

- temperatures: 203, 218, 228, 243, 273, 298 K.

Table 2 summarizes the uncertainties of BP data, and also shows type A/B breakdown. The relative standard measurement uncertainty of the BP ozone absorption cross section is stated to be around $\pm 1 \%$. It seems to be an underestimation, since BP relative spectra were scaled to the Hearn value at $253.65 \mathrm{~nm}$, which is reported with a $1.4 \%$ relative standard measurement uncertainty (see Sect. 3.1). Systematic uncertainties of the BP data are not discussed by Bass and Paur in their original publications (Bass and Paur, 1985; Paur and Bass, 1985).

\subsection{Uncertainty budget of BDM}

Ozone absorption cross sections provided by Brion et al. (1993), Daumont et al. (1992), and Malicet et al. (1995) further extend the wavelength coverage (into the visible spectrum) compared to BP. The following information is available on the experimental details of BDM data in the HartleyHuggins ozone absorption band:

- wavelength range: 195-520 nm (except at $273 \mathrm{~K}: 300$ $520 \mathrm{~nm})$;

- spectral resolution: $0.01 \mathrm{~nm}$;

- wavelength grid: in steps of $0.01 \mathrm{~nm}$;

- concatenation: $15 \mathrm{~nm}$ wide spectral cuts, $5 \mathrm{~nm}$ overlap;

- number of spectra averaged: 10 ;

- temperatures: 218, 228, 243, 273, $295 \mathrm{~K}$;

- temperature uncertainty: from $0.05 \mathrm{~K}$ at $295 \mathrm{~K}$ to $0.3 \mathrm{~K}$ at $218 \mathrm{~K}$; 
Table 1. Summary of uncertainties as reported by Hearn (1961).

\begin{tabular}{|c|c|c|c|c|}
\hline \multicolumn{5}{|c|}{ Random errors } \\
\hline $\begin{array}{l}\text { Wavelength uncertainty at } \\
253.65 \mathrm{~nm}\end{array}$ & $\begin{array}{l}\text { rms deviation } \\
\text { (mean of } 6 \text { observations) }\end{array}$ & $\begin{array}{l}\text { Absorption length } \\
(0.744 \mathrm{~cm})\end{array}$ & Pressure & $\begin{array}{l}\text { Total SD } \\
(\mathrm{rms})\end{array}$ \\
\hline Type B & Type A & \multicolumn{3}{|c|}{ Type B } \\
\hline $0.09 \mathrm{~nm}$ & $1.05 \%$ & $0.54 \%$ & $0.81 \%$ & $1.4 \%$ \\
\hline \multicolumn{5}{|c|}{ Systematic errors } \\
\hline $\begin{array}{l}\text { Wavelength uncertainty } \\
\text { at } 253.65 \mathrm{~nm}\end{array}$ & Correction for stray light & \multicolumn{3}{|c|}{ Correction for companion } \\
\hline $0.09 \mathrm{~nm}$ & 0.0 & \multicolumn{3}{|c|}{-} \\
\hline \multicolumn{5}{|c|}{ Best estimates of the absorption coefficients } \\
\hline $\begin{array}{l}\text { Wavelength uncertainty } \\
\text { at } 253.65 \mathrm{~nm}\end{array}$ & \multicolumn{4}{|c|}{ Molecular absorption cross section } \\
\hline $0.09 \mathrm{~nm}$ & \multicolumn{4}{|c|}{$114.7 \pm 2.4 \times 10^{-19} \mathrm{~cm}^{2}$} \\
\hline
\end{tabular}

Table 2. Absolute uncertainties reported by Bass and Paur (1985) and Paur and Bass (1985).

\begin{tabular}{|c|c|c|}
\hline Type & Uncertainty & Values \\
\hline A & Uncertainty in the transmittance determination & 2 in $10^{5}$ (arising from counting statistics) \\
\hline \multirow{5}{*}{ B } & Wavelength uncertainty & $0.025 \mathrm{~nm}$ \\
\hline & Sample temperature stability & better than $1 \mathrm{~K}$ \\
\hline & Temperature uncertainty & $0.25 \mathrm{~K}$ \\
\hline & Pressure measurement uncertainty & $1 \mathrm{mbar}$ \\
\hline & Absolute scaling & using the value of Hearn at $253.65 \mathrm{~nm}$ \\
\hline
\end{tabular}

- light source reference spectra: recorded before and after the ozone spectra;

- absolute scaling: measurements of total pressure.

Table 3 provides the original notation of the uncertainty budget of BDM data, and also shows Type A/Type B breakdown. The information on the relative standard measurement uncertainty of the BDM ozone absorption cross section is wavelength-dependent (see last row of Table 3).

\subsection{Uncertainty budget of SG}

Ozone absorption cross sections reported by Serdyuchenko et al. (2014) were obtained for 11 temperatures in a wide spectral range using two spectrometers (Fourier transform and Echelle grating spectrometers). Tables 4 and 5 summarize the information on the experimental details and uncertainties of the SG cross-section data.

In the $213-350 \mathrm{~nm}$ wavelength region, the relative standard measurement uncertainty of the SG ozone absorption cross section is wavelength-dependent and ranges between 1 and $3 \%$. The dominating uncertainty source is the statistical repeatability of the spectral measurements, influenced by stability of the light source and detector noise. These two factors have a greater impact when the intensities of the spectra contributing to the OD calculation either differ greatly (strong absorption, close to saturation) or are very close to each other. This effect is demonstrated in Fig. 1, showing the concatenated OD spectrum and relative uncertainties of the corresponding constituent spectral cuts. The latter were calculated according to the law of propagation of uncertainty using standard deviations and mean values of $\mathrm{I}$ and $\mathrm{I}_{0}$ spectra, respectively, which determine the optical density OD (see Eq. 3).

Table 6 summarizes the uncertainties for all cross sections' datasets discussed here.

\section{Temperature dependence and uncertainties}

In general the ozone cross sections were determined at selected atmospherically relevant temperatures. BP and BDM data encompass six and five temperatures, respectively, while $\mathrm{SG}$ is available at eleven temperatures. The original data can be obtained from http://satellite.mpic.de/spectral_atlas/ cross_sections/Ozone/O3.spc. Table 7 summarizes the available temperatures for all three datasets. 
Table 3. Summary of uncertainties as reported by Daumont et al. (1992) and Malicet et al. (1995).

\begin{tabular}{llll}
\hline & & Daumont et al. (1992) & Malicet et al. (1995) \\
\hline Type & Quantity & Uncertainty & Uncertainty \\
\hline A & $\begin{array}{l}\text { Absorbance } \\
\text { for } \lambda<335 \mathrm{~nm})\end{array}$ & $1 \%$ & $1 \%$ \\
& Optical path & $0.05 \%$ & \\
\hline & Ozone pressure & $0.1 \%$ & $0.05 \%$ \\
Impurities & $<0.1 \%$ & $0.1 \%$ \\
Temperature & $0.02 \%$ & $<0.1 \%$ \\
& & & from $0.02 \%$ at $295 \mathrm{~K}$ \\
& Wavelength & $<0.05 \%$ (Hartley band) & up to $0.15 \%$ at $218 \mathrm{~K}$ \\
& & & \\
\hline & Total (systematic) error & $1.3 \%$ (Hartley band) & $1.3-1.5 \%$ (Hartley band) \\
& Random error (rms) & $1.3-2.5 \%$ (Huggins band) & $1.3-3.5 \%$ (Huggins band) \\
& (for $\lambda<340$ nm) & $0.9-2.2 \%$ & $0.3-2.0 \%$ \\
\hline
\end{tabular}

Table 4. Experimental details and statistical uncertainty of OD spectra for different wavelength regions for SG data (Serdyuchenko et al., 2014). FTS denotes the Fourier transform spectrometer. The detector types used are silicon (Si) and germanium phosphorus (GaP) photodiodes, as well as an intensified charge-coupled device (ICDD).

\begin{tabular}{lllllll}
\hline $\begin{array}{l}\text { Spectral } \\
\text { region } \\
(\mathrm{nm})\end{array}$ & $\begin{array}{l}\text { Spectrometer, } \\
\text { detector }\end{array}$ & $\begin{array}{l}\text { Resolution } \\
(\mathrm{nm})\end{array}$ & Calibration & $\begin{array}{l}\text { Path } \\
(\mathrm{cm})\end{array}$ & $\begin{array}{l}\text { Lamp } \\
\text { stability* } \\
(\%)\end{array}$ & $\begin{array}{l}\text { Optical } \\
\text { density }\end{array}$ \\
\hline $213-310$ & Echelle, ICCD & 0.018 & Relative & 5 & $D_{2}, 0.5$ & $0.5-2$ \\
$310-335$ & FTS, GaP & 0.01 & Absolute & 135 & $\mathrm{Xe}, 2$ & $0.1-2$ \\
$335-350$ & FTS, GaP & 0.012 & Relative & 270 & $\mathrm{Xe}, 1$ & $0.1-1$ \\
$350-450$ & Echelle, ICCD & 0.02 & Relative & $\sim 2000$ & $\mathrm{Xe}, 1$ & $0.05-1$ \\
$450-780$ & FTS, Si & $0.02-0.06$ & Absolute & 270 & $\mathrm{~W}, 0.2$ & $0.05-2$ \\
$780-1100$ & FTS, Si & $0.12-0.24$ & Relative & 270 & $\mathrm{~W}, 0.2$ & $0.001-0.1$ \\
\hline
\end{tabular}

* during the entire measurement $D_{2}, \mathrm{Xe}$ - deuterium and xenon discharge lamps, $\mathrm{W}$ - tungsten filament lamp.

The temperature dependence of the ozone absorption cross sections is commonly described by the so-called Bass-Paur parameterization (Paur and Bass, 1985), which is a quadratic polynomial:

$\sigma_{p}(\lambda, T)=a_{0}+a_{1} t+a_{2} t^{2}$.

The temperature coefficients $a_{0}, a_{1}$, and $a_{2}$ are determined in a multivariate linear regression using the cross-section data $\sigma\left(\lambda, t_{i}\right)$ measured at selected temperatures $t_{i}$. They were calculated in the wavelength range 290-360 nm (BP: up to about $338 \mathrm{~nm}$ ), which is the spectral range with the highest temperature sensitivity (Huggins ozone band). The temperature $t$ in Eq. (4) is given in degrees Celsius $(t=T-273.15 \mathrm{~K})$. The uncertainty of the calculated cross section at a given temperature is then given by

$$
\Delta \sigma_{p}=\sqrt{\left(\Delta a_{0}\right)^{2}+\left(\Delta a_{1}\right)^{2} t^{2}+\left(\Delta a_{2}\right)^{2} t^{4}} .
$$

The $228 \mathrm{~K}$ temperature data of BP have been excluded from the polynomial fit as there is a gap between 295 and
$304 \mathrm{~nm}$. Liu et al. (2007) noted a systematic bias in the $273 \mathrm{~K}$ BDM data and reported better ozone retrieval fit results if this temperature is excluded. These temperature data also do not provide data below $300 \mathrm{~nm}$. As noted by Orphal and Chance (2003) and Weber et al. (2013), there is a systematic wavelength shift between BP and BDM. Shifting the BP data by $+0.029 \mathrm{~nm}$ leads to better agreement (to within $0.5 \%$ ) between BDM and BP (Weber et al., 2013). The SG data wavelength scale agrees to within uncertainties with BDM (Gorshelev et al., 2014).

Figures 2 to 4 show the temperature coefficients including the $1 \sigma$ uncertainties (see Eq. 5) for the BP, BDM, and SG data, respectively. As the SG data are somewhat noisy near $300 \mathrm{~nm}$, in the July 2013 version of the SG data, a fast Fourier transform filter was applied in the spectral range 213-317 $\mathrm{nm}$. These figures also show that the BP data appear somewhat noisier than the others, and one striking difference between SG and BDM is the apparent bump in the third coefficient (blue line) near $305 \mathrm{~nm}$, evident in BDM. 
Table 5. Summary of absolute and relative measurement uncertainties for SG dataset.

\begin{tabular}{llll}
\hline Systematic uncertainties (abs) & $(\%)$ & Random uncertainties (abs) & $(\%)$ \\
\hline Ozone impurity: & 0.005 & Ozone initial pressure & $<1$ \\
oxygen impurity leaks & $<0.1$ & Pressure fluctuations $(<0.04 \mathrm{mb})$ & $<0.08$ \\
Pressure sensors $(0.02 \mathrm{mb})$ & 0.04 & Temperature fluctuations $(<0.3 \mathrm{~K})$ & \\
Temp. sensors' offset $(1 \mathrm{~K})$ & $0.3-0.5$ & Light source stability, relative to optical den- & $<0.1$ \\
Temp. nonuniformity (1 K) & $0.3-0.5$ & sity OD $=1$ (depending on spectral region) & $0.2-2$ \\
Cell length $(0.1-1.0 \mathrm{~mm})$ & $0.04-0.07$ & & \\
\hline
\end{tabular}

Combined standard relative uncertainty

(excluding low absorption regions near 380nm and above $800 \mathrm{~nm}$ )

\begin{tabular}{rr}
\hline $0.4-0.7$ & $1-2.2$ \\
\hline
\end{tabular}

Table 6. Summary of uncertainties for Hearn, BP, BDM, and SG ozone cross sections.

\begin{tabular}{lllll}
\hline Dataset & $\begin{array}{l}\text { Scaling } \\
\text { method }\end{array}$ & $\begin{array}{l}\text { Random } \\
\text { (statistical) }\end{array}$ & Systematic & $\begin{array}{l}\text { Relative standard } \\
\text { measurement } \\
\text { uncertainty (\%) }\end{array}$ \\
\hline Hearn $(253.65 \mathrm{~nm})$ & Absolute, pure ozone & 1.05 & - & 1.4 \\
$\mathrm{BP}$ & Using Hearn & 1 & 2.1 & $>2.1$ \\
$\mathrm{BDM}$ & Absolute, pure ozone & $0.9-2.2$ & 1.3 (Hartley) & $2-3$ \\
& & & $1.3-3.5$ (Huggins) & $2-4$ \\
$\mathrm{SG}$ & Absolute, pure ozone & $1-2.2$ & $0.4-1.7$ & $1.1-3$ \\
\hline
\end{tabular}

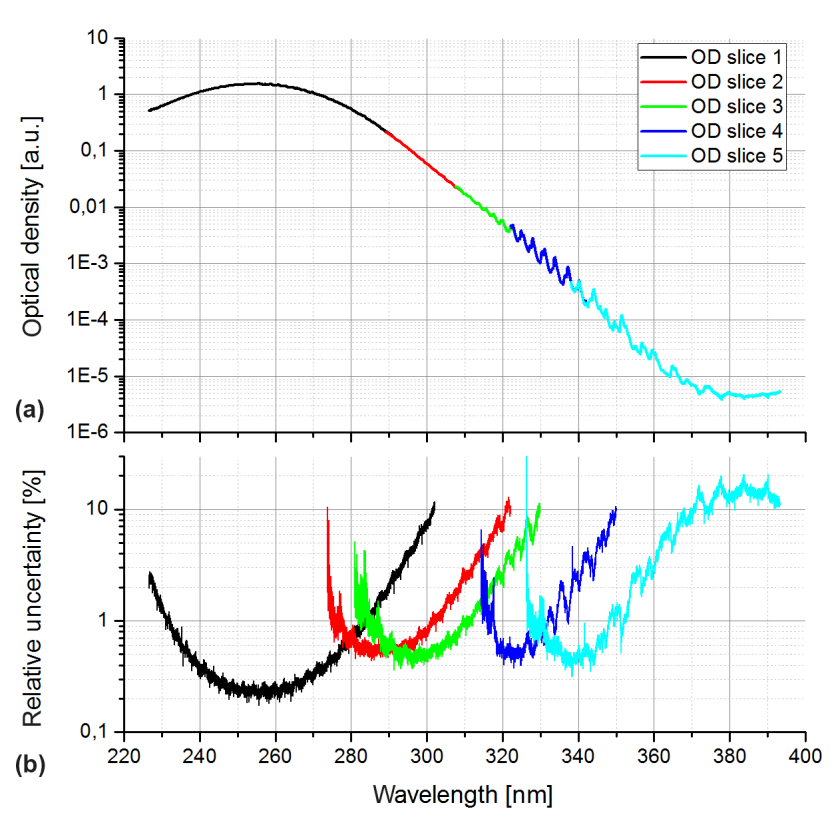

Figure 1. Upper panel: concatenated optical density spectrum. Lower panel: relative uncertainty of various OD spectra used for concatenation. Instruments: Echelle/Fourier transform spectrometer (FTS); number of averaged spectra: 2000 (Echelle)/100 (FTS); acquisition time: $\sim 30 \mathrm{~min}$; light sources: $\mathrm{Xe}$ and $D_{2}$ lamps. From Serdyuchenko et al. (2014).

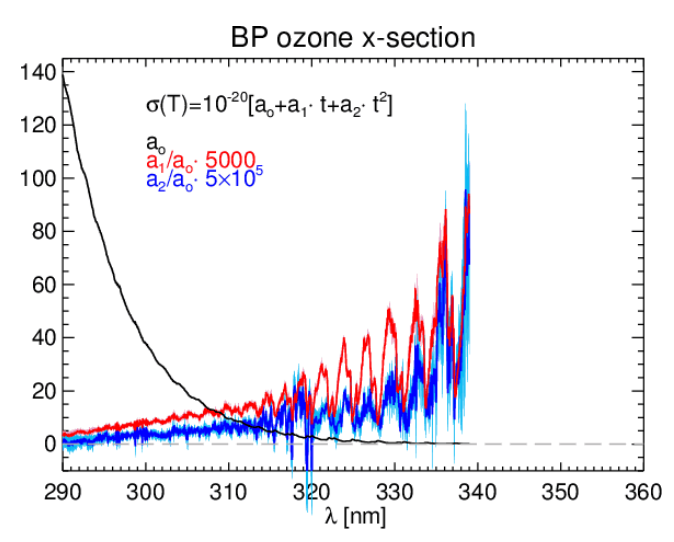

Figure 2. Temperature coefficients and their uncertainty $(1 \sigma)$ of the $\mathrm{BP}$ data as a function of wavelength $\lambda$. For some coefficients and/or selected wavelengths, the uncertainties are too small to be visible.

Figure 5 shows the uncertainty from the polynomial fit as a function of wavelength for $T=193$ and $227 \mathrm{~K}$. BP data uncertainties get fairly large above $330 \mathrm{~nm}$, reaching nearly $25 \%$ for some wavelengths at $220 \mathrm{~K}$ and more than $60 \%$ at $193 \mathrm{~K}$. The uncertainty of the BDM data ranges between 0 and $2 \%$ up to $330 \mathrm{~nm}$, while SG data show a fairly constant uncertainty of about $1 \%$ on average at $T=227 \mathrm{~K}$. The uncertainties are doubled at the lower temperature. The very low uncertainty for BP and BDM at some wavelength is mainly due to the very low number of available tempera- 
Table 7. Available temperatures for various ozone absorption cross-section data. Temperatures provided in brackets were not used to determine temperature coefficients since they did not cover the complete wavelength range of $290-340 \mathrm{~nm}$.

\begin{tabular}{lll}
\hline $\begin{array}{l}\text { Ozone absorption } \\
\text { cross section }\end{array}$ & $\begin{array}{l}\text { Temperatures } \\
(\mathrm{K})\end{array}$ & $\begin{array}{l}\text { Wavelength } \\
\text { range (nm) }\end{array}$ \\
\hline BP, Paur and Bass (1985) & $203,218,(228), 243,273,298$ & $245-343$ \\
$\begin{array}{l}\text { BDM, Malicet et al. (1995) } \\
\text { SG, Serdyuchenko et al. (2014) }\end{array}$ & $218,228,243,(273), 295$ & $195-520$ \\
\hline
\end{tabular}

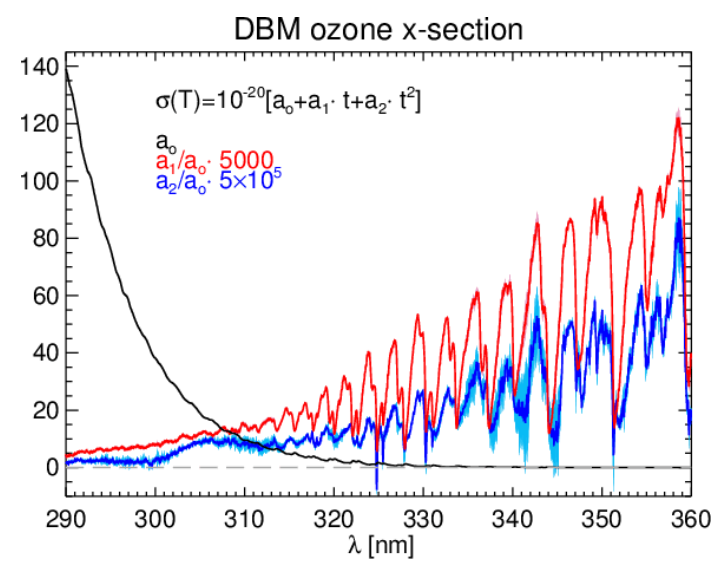

Figure 3. Same as Fig. 2, but for BDM data.

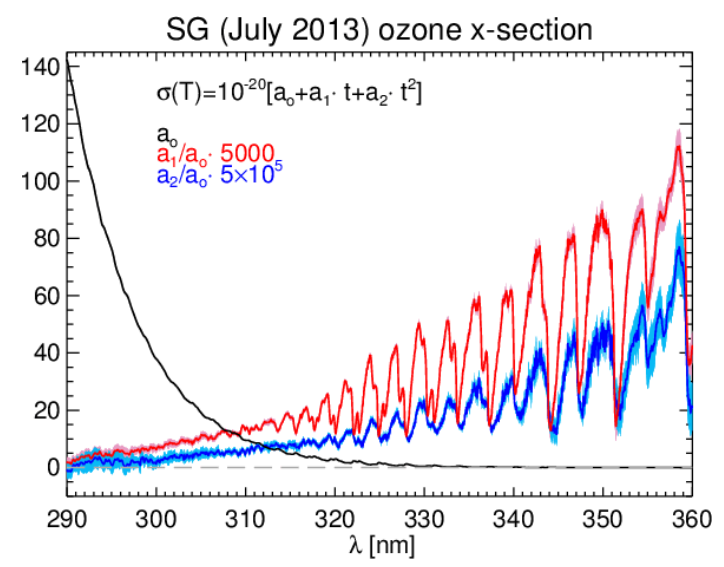

Figure 4. Same as Fig. 2, but for SG (July 2013 version) data.

tures (4-5) that leads, in some cases, to overfitting of the data with a quadratic polynomial.

The spectral resolution of the three datasets varies from $0.01 \mathrm{~nm}(\mathrm{BDM})$ to $0.05 \mathrm{~nm}(\mathrm{BP})$. In order to determine the ozone cross section at a specific instrument resolution, one can either convolve all the various temperature data with the instrument function and then apply the polynomial fit, or the coefficient spectra (as shown in Figs. 2-4) are convolved and the polynomial coefficients from the original data are used.

\section{Overall uncertainty: Monte Carlo simulation}

The uncertainty given in Eq. (5) reflects only the uncertainty from the temperature parameterization using a polynomial (if we assume that a quadratic dependence in temperature is true), thus excluding the experimental uncertainties as discussed in Sect. 3. One main motivation to only show the uncertainties arising from the polynomial fit is to demonstrate that with only few temperatures available for some of the datasets (BP, BDM), the uncertainty in the temperature dependence is strongly underestimated due to overfitting. In order to estimate the overall uncertainty including uncertainties from measurements (random and systematic), wavelength registration, and the temperature parameterization, an extensive Monte Carlo simulation (JCGM-101, 2008) was carried out.

Table 8 summarizes the uncertainties simulated. The numbers are mainly based upon the uncertainty as reported in Table 6. It was assumed that the probability density function (PDF) is Gaussian for all uncertainties.

The values used here are the minimum uncertainties as summarized in Table 6; however, it should be noted that the (random) measurement uncertainties vary with wavelengths, but this is neglected here for all datasets as the exact wavelength dependence is not known for most of the data (no information provided for BP, only range of values given for BDM). The systematic uncertainty for each temperature dataset of $\mathrm{BP}$ was set to $2 \%$, which reflects the potential bias of up to $+2 \%$ of the Hearn value (used to scale the BP data) with respect to the more recent and very accurate mercury line measurements by Viallon et al. (2015); see Sect. 3.1.

Uncertainties for all parameters in Table 8 are drawn from a Gaussian random generator to perturb the cross-section data at each available temperature. Random uncertainties means that for each temperature available, a new set of random perturbations were calculated, while systematic uncertainties means that uncertainties drawn from the random generator were applied to all temperature data simultaneously. A total of 10000 perturbed datasets of cross-section data were then generated and each fitted by a quadratic polynomial in temperature. The $1 \sigma$ distributions from the sample polynomials provided then the overall $1 \sigma$ uncertainty as a function of temperature. MC simulations were repeated for all wavelengths between 290 and $370 \mathrm{~nm}$ in steps of $0.01 \mathrm{~nm}$. Cross 

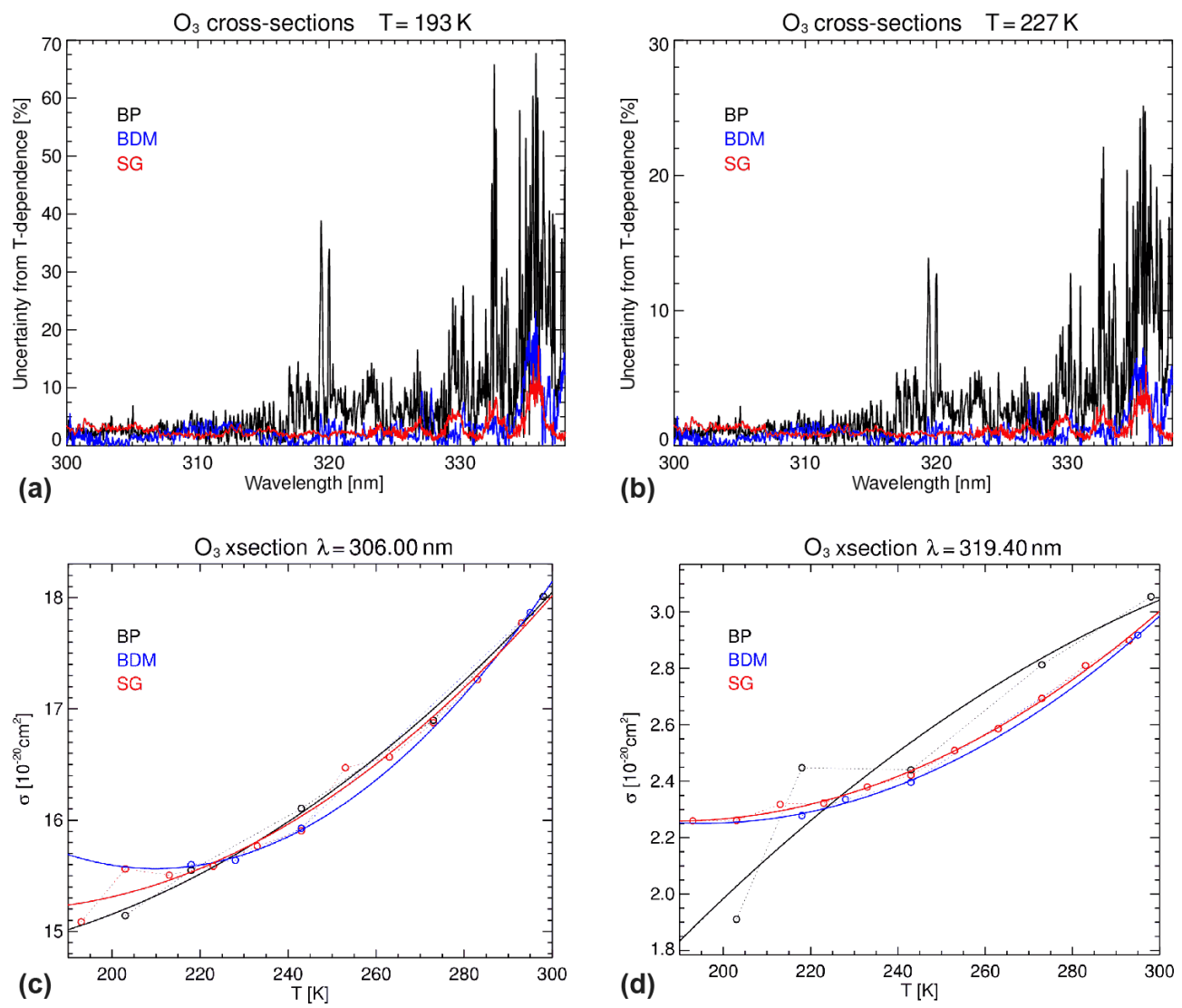

Figure 5. Panels (a) and (b): $1 \sigma$ uncertainty of BP, BDM, and SG ozone cross sections at $T=193$ and $227 \mathrm{~K}$, respectively, from the polynomial temperature fit (Eq. 5). Note the change in scale of the ordinate axes. Panels (c) and (d): measured ozone cross sections (points) and polynomial fit (solid lines) for BP, BDM, and SG at 306 and $319.4 \mathrm{~nm}$, respectively.

Table 8. Uncertainties accounted for in the Monte Carlo simulation assuming a Gaussian PDF.

\begin{tabular}{ll}
\hline Uncertainty type (290-370 nm) & Value $(1 \sigma)$ \\
\hline Cross section at each $T$ (random) & $1 \%$ \\
Cross section at each $T$ (systematic) & $1.3 \%{ }^{*}$ \\
Wavelength registration at each $T$ (random) & $0.005 \mathrm{~nm}$ \\
Wavelength registration at each $T$ (systematic) & $0 \mathrm{~nm}^{* *}$ \\
Temperature $T$ (random) & $0.5 \mathrm{~K}$ \\
Temperature $T$ (systematic) & $1 \mathrm{~K}$ \\
Polynomial in $T$ & combined resampling residuals and \\
& wild boot strap (normal distributed) \\
\hline
\end{tabular}

$* 2 \%$ for BP ozone cross sections. ${ }^{* *}$ It is assumed that wavelength shifts can be corrected in ozone retrievals (e.g., Coldewey-Egbers et al., 2005).

correlations between adjacent wavelengths, which are difficult to estimate, were neglected.

In order to estimate the effect of the measurement errors on uncertainties from the $T$ polynomial, a combination of the resampling residual and wild boot strap method was applied (Wu, 1986). The residuals from the polynomial fit are given by

$\varepsilon_{i}=\sigma\left(\lambda, t_{i}\right)-\sigma_{p}\left(\lambda, t_{i}\right)$, where $\sigma_{p}\left(\lambda, t_{i}\right)$ is the fitted polynomial (see Eq. 4) and $t_{i}$ are the selected temperatures for which the measured crosssection data $\sigma\left(\lambda, t_{i}\right)$ are available. In the Monte Carlo simulation, the residuals are distributed randomly to different temperatures as follows: 

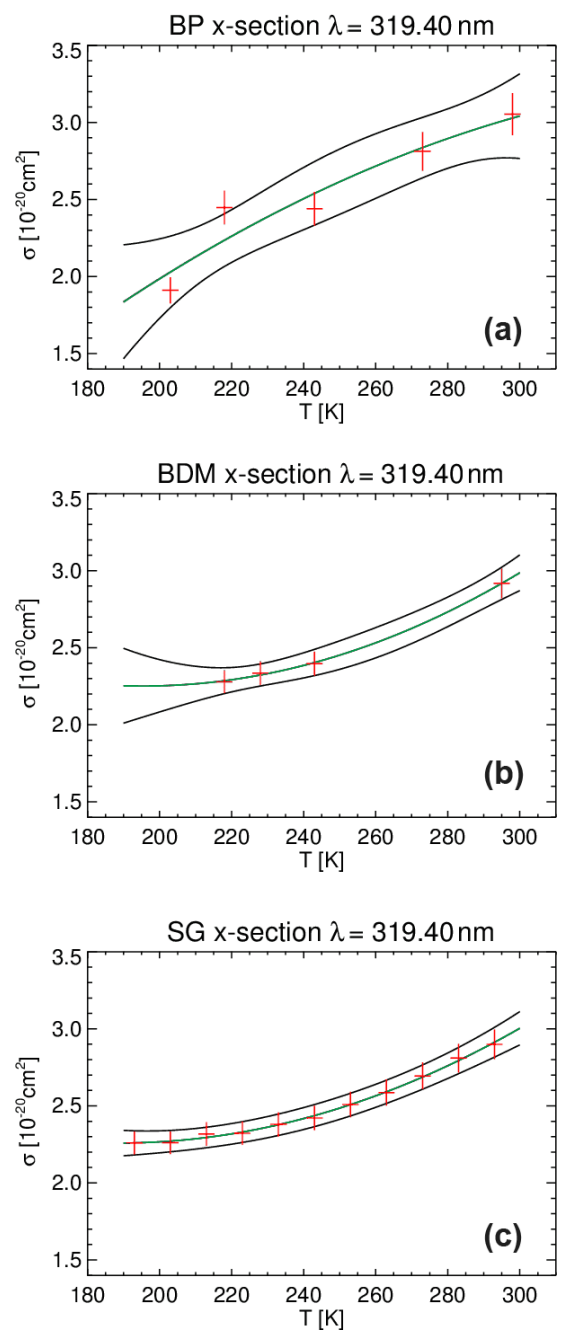

Figure 6. Modeled uncertainties of the three major ozone absorption cross sections (BP, SG, and BDM) at $319.4 \mathrm{~nm}$ based upon MC simulations. Red crosses show the measured data, including temperature and measurement uncertainties (here expressed as the square sum of random and systematic errors). The green curve is the fitted polynomial and the black curves show the modeled $\pm 2 \sigma$ uncertainty.

$\sigma^{\prime}\left(\lambda, t_{i}\right)=\sigma\left(\lambda, t_{i}\right)+\xi \cdot \epsilon_{j}$

in order to perturb the cross-section data. $\xi$ is a normal distributed random number with mean 0 and variance of 1 . The normally distributed random number generator used in the MC simulation is based upon the Box-Muller transform (Box and Muller, 1958). The total sample size of $10^{4}$ provides a reasonable compromise between computation time and precision of the simulation. For each wavelength between 290 and $370 \mathrm{~nm}(\mathrm{BP}: \sim 339 \mathrm{~nm})$ in steps of $0.01 \mathrm{~nm}$, the Monte Carlo simulation was carried out.

Figures 6 and 7 show the results from the MC simulation of uncertainties for the three major ozone cross-section data
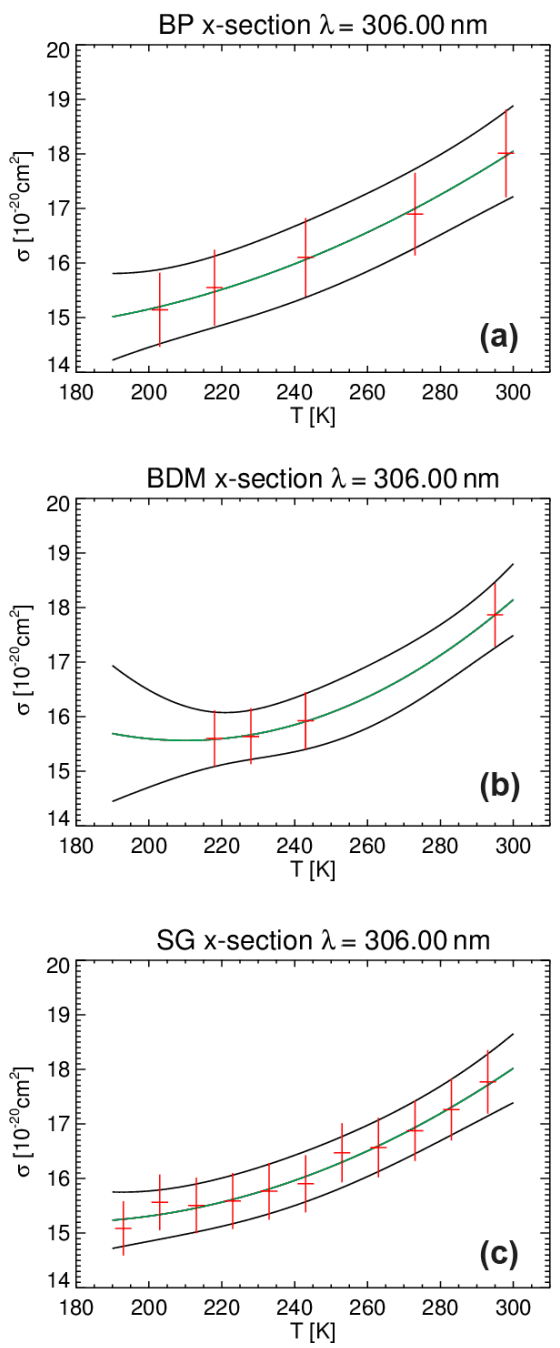

Figure 7. Same as Fig. 6, but at $306 \mathrm{~nm}$.

at 319.4 and $306 \mathrm{~nm}$, respectively, as an example. These plots correspond to the data shown in panels $\mathrm{c}$ and d of Fig. 5. The uncertainties are very similar for BDM and SG, except for the lowest temperatures $(T<215 \mathrm{~K})$ where BDM uncertainties increase due to the extrapolation of the fitted polynomial. The larger systematic measurement uncertainty of the BP data (2\%), due to the uncertainties related to the Hearn value at the mercury line used for scaling the BP data (see Sect. 3.1), leads to larger overall uncertainties in the BP data. The uncertainties from the laboratory measurements are indicated in Figs. 6 and 7 by the red crosses, which show the overall uncertainty from the laboratory measurements (systematic and random, vertical bar) as well as the temperature uncertainty (horizontal bar). The overall uncertainties from the MC simulation are generally in good agreement with measurement uncertainty estimates; however, Fig. 6a (BP at $319.4 \mathrm{~nm}$ ) clearly indicates that the overall uncertainty is larger here than the uncertainties from the measurements, and this is due to the outlier at $203 \mathrm{~K}$, which increases the 

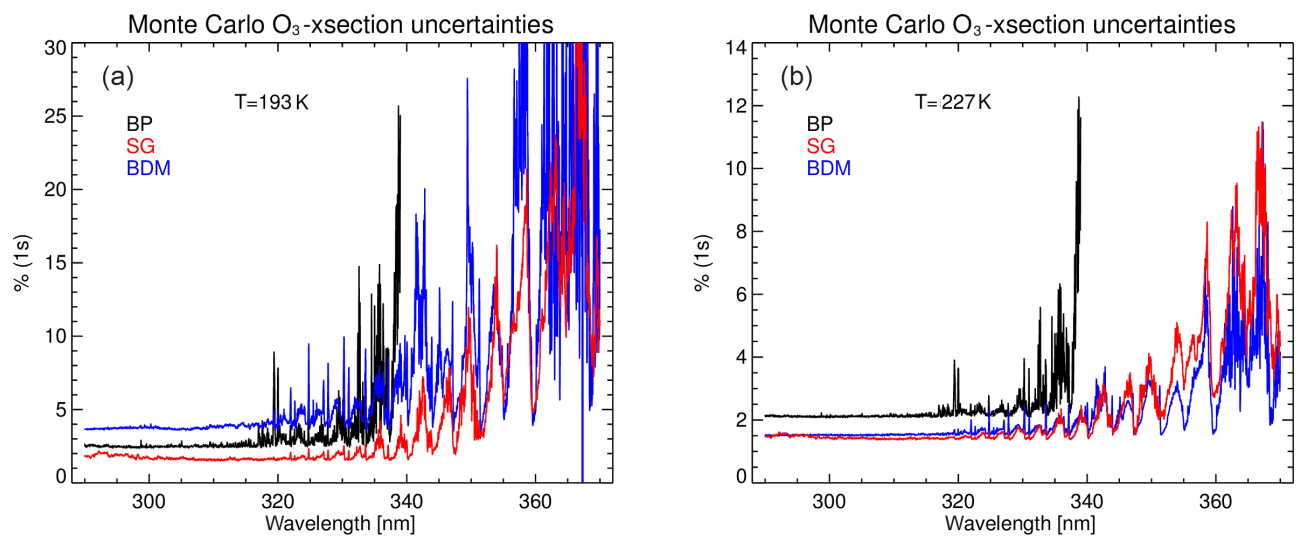

Figure 8. Uncertainty ( $1 \sigma$ ) of BP, BDM, and SG ozone cross sections at $T=193 \mathrm{~K}$ (left) and $227 \mathrm{~K}$ (right), respectively, from the MC simulation. Note the change in scale of the ordinate axes.

contribution from the uncertainty of the polynomial largely. Figure 8 shows the uncertainties as a function of wavelengths for selected temperatures. At $227 \mathrm{~K}$ the overall uncertainties are about $1.5 \%$ for both BDM and SG, while BP uncertainties are about $2.1 \%(1 \sigma)$. Above $330 \mathrm{~nm}$ the uncertainties increase for all datasets. At very low temperatures, e.g., $193 \mathrm{~K}$, the BDM uncertainties increase to about $4 \%(1 \sigma)$, while SG remains at $2 \%(1 \sigma)$. BP uncertainties are about $2.5 \%(1 \sigma)$ and are also lower than BDM. Similar to $227 \mathrm{~K}$, the uncertainties increase at the longest wavelengths. At temperatures above $215 \mathrm{~K}$, the uncertainties of BDM and SG are very similar; at lower temperatures the BDM uncertainties significantly increase due to the lack of very low temperature measurements.

\section{Summary and conclusion}

Realistic and comparable uncertainty budgets were derived from three major ozone absorption cross-section datasets that are used in various remote sensing applications. First a review of the published literature on the uncertainty of the BP, $\mathrm{BDM}$, and SG datasets was given. The uncertainties of these three datasets are summarized in Table 6 and are now directly comparable between the various datasets. For remote sensing application, in particular in the Huggins ozone band, the temperature dependence of the ozone cross sections has to be accounted for and this is typically done using a quadratic polynomial as a function of temperature. Using the updated uncertainty estimates from Table 6 and a residual boot strap method for estimating the uncertainties from the temperature polynomial, a Monte Carlo simulation was carried out. However, one should note that due to lack of information from the peer-review literature, the wavelength dependence of the uncertainties (see Table 6) was neglected in our simulations.

In the Huggins band the overall uncertainty of the temperature-dependent ozone cross section is about $1.5 \%$ $(1 \sigma)$ for BDM and SG and $2.1 \%(1 \sigma)$ for BP up to about
$330 \mathrm{~nm}$. At temperatures below about $215 \mathrm{~K}$ the uncertainties in the BDM data increase more strongly than for the others, as the lowest measured temperature for BDM is $218 \mathrm{~K}$ and the extrapolation of the polynomial leads to larger uncertainties. Above $330 \mathrm{~nm}$ the uncertainties increase significantly for all datasets. Ozone retrievals exploiting the UV spectral range usually focus on wavelengths below $335 \mathrm{~nm}$.

In recent years a seemingly larger proportion of publications have been adhering to the guidelines of uncertainty reporting as recommended by JCGM-100 (2008). However, there is still either a lack of consistency in the approaches used, or uncertainty budgets are not detailed enough. It is especially challenging to re-evaluate uncertainty budgets of older published datasets. In this paper we attempted to provide a more realistic uncertainty budget that may be useful when trying to establish the contribution from ozone absorption cross sections to the overall uncertainty of retrieved ozone. This work is part of a project on the traceability of total ozone measurements (ATMOZ), where we plan several applications of our results in simulated retrievals as well as the general impact on satellite and ground retrievals of total ozone. This will be subject of other publications.

\section{Data availability}

The temperature coefficients for the three major crosssection data with uncertainties from the MC simulations (Weber, 2016) are available at http://www.iup.uni-bremen. de/UVSAT/datasets/uv-ozone-absorption-cross-sections.

Acknowledgements. This work was supported in part by a grant from EMRP within the ENV59-ATMOZ ("Traceability for atmospheric total column ozone") Joint Research Programme (JRP), University of Bremen, and the State of Bremen. The EMRP is jointly funded by the EMRP-participating countries within EURAMET and the European Union. 
The article processing charges for this open-access publication were covered by the University of Bremen.

Edited by: M. Van Roozendael

Reviewed by: two anonymous referees

\section{References}

ACSO: WMO/GAW-IO3C-IGACO-O3 Activity "Absorption Cross Sections of Ozone (ACSO)", available at: http://igaco-o3.fmi.fi/ ACSO/ (last access: November 2015), 2010.

Bass, A. M. and Paur, R. J.: The ultraviolet cross-sections of ozone: I. the measurements, in: Atmospheric Ozone, edited by: Zerefos, C. S., and Ghazi, A., Proc. Quadrennial Ozone Symposium, Halkidiki, Greece, 1984, Reidel, D., Dordrecht, 606-610, 1985.

Box, G. E. P. and Muller, M. E.: A note on the generation of random normal deviates, Ann. Math. Stat., 29, 610-611, doi:10.1214/aoms/1177706645, 1958.

Brion, J., Chakir, A., Daumont, D., Malicet, J., and Parisse, C.: High-resolution laboratory absorption cross section of $\mathrm{O}_{3}$. Temperature effect, Chem. Phys. Lett., 213, 610-612, doi:10.1016/0009-2614(93)89169-I, 1993.

Chehade, W., Gür, B., Spietz, P., Gorshelev, V., Serdyuchenko, A., Burrows, J. P., and Weber, M.: Temperature dependent ozone absorption cross section spectra measured with the GOME-2 FM3 spectrometer and first application in satellite retrievals, Atmos. Meas. Tech., 6, 1623-1632, doi:10.5194/amt-6-1623-2013, 2013.

Chehade, W., Gorshelev, V., Serdyuchenko, A., Burrows, J. P., and Weber, M.: Revised temperature-dependent ozone absorption cross-section spectra (Bogumil et al.) measured with the SCIAMACHY satellite spectrometer, Atmos. Meas. Tech., 6, 30553065, doi:10.5194/amt-6-3055-2013, 2013.

Coldewey-Egbers, M., Weber, M., Lamsal, L. N., de Beek, R., Buchwitz, M., and Burrows, J. P.: Total ozone retrieval from GOME UV spectral data using the weighting function DOAS approach, Atmos. Chem. Phys., 5, 1015-1025, doi:10.5194/acp5-1015-2005, 2005.

Daumont, D., Brion, J., Charbonnier, J., and Malicet, J.: Ozone UV spectroscopy I: Absorption cross-sections at room temperature, J. Atmos. Chem., 15, 145-155, doi:10.1007/BF00053756, 1992.

Gorshelev, V., Serdyuchenko, A., Weber, M., Chehade, W., and Burrows, J. P.: High spectral resolution ozone absorption crosssections - Part 1: Measurements, data analysis and comparison with previous measurements around $293 \mathrm{~K}$, Atmos. Meas. Tech., 7, 609-624, doi:10.5194/amt-7-609-2014, 2014.

JCGM-100: Evaluation of measurement data - Guide to the expression of uncertainty in measurement (JCGM-100), http://www.bipm.org/utils/common/documents/jcgm/JCGM_ 100_2008_E.pdf, (last access: November 2015), 2008

JCGM-101: Evaluation of measurement data - Supplement 1 to the "Guide to the expression of uncertainty in measurement" - Propagation of distributions using a Monte Carlo method (JCGM-101), http://www.bipm.org/utils/common/ documents/jcgm/JCGM_101_2008_E.pdf (last access: October 2015), 2008.
Hearn, A. G.: The absorption of ozone in the ultra-violet and visible regions of the spectrum, Proc. Phys. Soc., 78, 932-940, doi:10.1088/0370-1328/78/5/340, 1961.

Liu, X., Chance, K., Sioris, C. E., and Kurosu, T. P.: Impact of using different ozone cross sections on ozone profile retrievals from Global Ozone Monitoring Experiment (GOME) ultraviolet measurements, Atmos. Chem. Phys., 7, 3571-3578, doi:10.5194/acp7-3571-2007, 2007.

Malicet, J., Daumont, D., Charbonnier, J., Parisse, C., Chakir, A., and Brion, J.: Ozone UV spectroscopy. II. Absorption crosssections and temperature dependence, J. Atmos. Chem., 21, 263 273, doi:10.1007/BF00696758, 1995.

Orphal, J. and Chance, K.: Ultraviolet and visible absorption crosssections for HITRAN, J. Quant. Spectrosc. Ra., 82, 491-504, doi:10.1016/S0022-4073(03)00173-0, 2003.

Orphal, J., Staehelin, J., Tamminen, J., Braathen, G., De Backer, M.-R., Bais, A., Balis, D., Barbe, A., Bhartia, P. K., Birk, M., Burkholder, J. B., Chance, K., von Clarmann, T., Cox, A., Degenstein, D., Evans, R., Flaud, J.-M., Flittner, D., GodinBeekmann, S., Gorshelev, V., Gratien, A., Hare, E., Janssen, C., Kyrölä, E., McElroy, T., McPeters, R., Pastel, M., Petersen, M., Petropavlovskikh, I., Picquet-Varrault, B., Pitts, M., Labow, G., Rotger-Languereau, M., Leblanc, T., Lerot, C., Liu, X., Moussay, P., Redondas, A., Van Roozendael, M., Sander, S. P., Schneider, M., Serdyuchenko, A., Veefkind, P., Viallon, J., Viatte, C., Wagner, G., Weber, M., Wielgosz, R. I., and Zehner, C.: Absorption cross-sections of ozone in the ultraviolet and visible spectral regions: Status report 2015, J. Mol. Spectrosc., 327, 105-121, doi:10.1016/j.jms.2016.07.007, 2016.

Paur, R. J. and Bass, A. M.: The ultraviolet cross-sections of ozone: II. Results and temperature dependence, in: Atmospheric Ozone, edited by: Zerefos, C. S., and Ghazi A., Proc. Quadrennial Ozone Symposium, Halkidiki, Greece, 1984, Reidel, D., Dordrecht, 611-615, 1985.

Redondas, A., Evans, R., Stuebi, R., Köhler, U., and Weber, M.: Evaluation of the use of five laboratory-determined ozone absorption cross sections in Brewer and Dobson retrieval algorithms, Atmos. Chem. Phys., 14, 1635-1648, doi:10.5194/acp14-1635-2014, 2014.

Serdyuchenko, A., Gorshelev, V., Weber, M., and Burrows, J. P.: New broadband high-resolution ozone absorption cross-sections, Spectroscopy Europe, 23, 14-17, 2011.

Serdyuchenko, A., Gorshelev, V., Weber, M., Chehade, W., and Burrows, J. P.: High spectral resolution ozone absorption crosssections - Part 2: Temperature dependence, Atmos. Meas. Tech., 7, 625-636, doi:10.5194/amt-7-625-2014, 2014.

Sofen, E. D., Evans, M. J., and Lewis, A. C.: Updated ozone absorption cross section will reduce air quality compliance, Atmos. Chem. Phys., 15, 13627-13632, doi:10.5194/acp-1513627-2015, 2015.

Viallon, J., Lee, S., Moussay, P., Tworek, K., Petersen, M., and Wielgosz, R. I.: Accurate measurements of ozone absorption cross-sections in the Hartley band, Atmos. Meas. Tech., 8, 12451257, doi:10.5194/amt-8-1245-2015, 2015.

Weber, M., Chehade, W., Gorshelev, V., Serdyuchenko, A., Spietz, P.: Impact of ozone cross-section choice on WFDOAS total ozone retrieval applied to GOME, SCIAMACHY, and GOME-2 (1995-present), Technical Note Issue 2 with updates from November 2013, a contribution to IGACO- 
O3/ACSO, 2013, available at: http://www.iup.uni-bremen.de/ UVSAT_material/technotes/weber_acso_201311.pdf (last access: November 2015), 2013.

Weber, M.: UV ozone absorption cross-sections, available at: http://www.iup.uni-bremen.de/UVSAT/datasets/ uv-ozone-absorption-cross-sections, last access: 6 September 2016.

WMO-GAW: Absorption Cross-Sections of Ozone (ACSO) Status Report June 2015, WMO-GAW Report 218, World Meteorological Organization, Geneva, Switzerland, available at: http://www.wmo.int/pages/prog/arep/gaw/documents/FINAL_ GAW_218.pdf (last access: Septmeber 2016), 2015.
Wu, C. F. J.: Jackknife, bootstrap and other resampling methods in regression analysis (with discussions), Ann. Stat. 14, 1261-1350, doi:10.1214/aos/1176350142, 1986. 